\title{
How should we monitor pediatric patients with Duchenne muscular dystrophy? -A case report-
}

\author{
Hyun Ju Jung, Jong Bun Kim, Kyong Shil Im, Jeoung Hyuk Lee, Duk Ju Kim, Sung Ah Cho, and \\ Jae Myeong Lee \\ Department of Anesthesiology and Pain Medicine, The Catholic University of Korea College of Medicine, Seoul, Korea
}

Duchenne muscular dystrophy is a hereditary disorder characterized by progressive muscle weakness and contracture, and special care during anesthesia is needed in these patients. Because inhalational anesthetics and succinylcholine can cause fatal results, intravenous anesthetics are commonly used. However, monitorings for the pediatric population are not otherwise specified. We report our experience of a 6 year-old boy that underwent muscle biopsy suspicious of muscle dystrophy under general anesthesia. The patient received midazolam, fentanyl, propofol and a small dose of rocuronium. He was monitored with bispectral index (BIS), acceleromyography (TOF). At the end of surgery, recovery of TOF ratio to $90 \%$ was evaluated, followed by injection of pyridostigmine and glycopyrrolate. When reversal of neuromuscular block was confirmed quantitatively and clinically, the patient was extubated and he experienced no complication. (Korean J Anesthesiol 2011; 61: 159-161)

Key Words: Anesthesia, Bispectral index, Monitoring, Muscular dystrophy.

Anesthesia for children with Duchenne muscular dystrophy (DMD) should take into account rhabdomyolysis, malignant hyperthermia-like reaction and cardiac arrest. Intravenous and non-depolarizing muscle relaxants are commonly used in children. Inhalational anesthetics and succinylcholine can lead to complications. However, monitoring for pediatric patients are not otherwise specified.

Intravenous anesthesia with target-controlled infusion of propofol and remifentanil are limited by body weight and age. For young children, intravenous anesthesia is guided by vital signs, as neuromuscular monitoring for the pediatric population is not well established. We herein report our experience performing anesthetic monitoring on a child with suspected DMD.

\section{Case Report}

A 6 -year-old, $19 \mathrm{~kg}$, child was admitted to our hospital with

Received: October 1, 2010. Revised: February 9, 2011. Accepted: February 22, 2011.

Corresponding author: Jae Myeong Lee, M.D., Department of Anesthesiology and Pain Medicine, Uijeongbu St. Mary's Hospital, The Catholic University of Korea, 65-1, Geumo-dong, Uijeongbu 480-130, Korea. Tel: 82-31-820-3087, Fax: 82-31-821-8449, E-mail: jaemng@catholic.ac.kr (c) This is an open-access article distributed under the terms of the Creative Commons Attribution Non-Commercial License (http:// creativecommons.org/licenses/by-nc/3.0/), which permits unrestricted non-commercial use, distribution, and reproduction in any medium, provided the original work is properly cited. 
symptoms of proximal muscle weakness and a one-month history of difficulty climbing steps. He was planned to undergo muscle biopsy under the suspicion of DMD. Preoperative laboratory tests were abnormal: aspartate transaminase (AST) $560 \mathrm{IU} / \mathrm{L}$, alanine transaminase (ALT) $773 \mathrm{IU} / \mathrm{L}$, alkaline phosphatase $352 \mathrm{IU} / \mathrm{L}$, lactate dehydrogenase (LDH) 4,280 IU/L, creatinine phosphokinase (CPK) 32,730 IU/L. His electrolytes, urine exam, electrocardiography and chest radiography were unremarkable. Preoperative echocardiography and pulmonary function test were considered to be unnecessary by the attending pediatrician.

The patient received no premedication. Upon arrival in the operating room, standard monitoring, including EKG, pulse oximetry, end-tidal capnography, transesophageal temperature probe, bispectral index (BIS vista ${ }^{\mathrm{TM}}$, Aspect Medical Systems, The Netherlands) and Train-of-four monitor (TOF watch ${ }^{\mathrm{TM}}$, Organon, Ireland) were applied.

Anesthesia was induced with intravenous midazolam 1 mg, fentanyl $25 \mu \mathrm{g}$, and continuous infusion of propofol. Rocuronium bromide $6 \mathrm{mg}$ was administered to facilitate orotracheal intubation. BIS 40-65 was maintained and $30 \mathrm{~mA}$ at $2 \mathrm{~Hz}$ TOF was monitored during anesthesia. Anesthesia was maintained with nitrous oxide $50 \%$ in oxygen and end-tidal $\mathrm{CO}_{2}$ was maintained at $30-35 \mathrm{mmHg}$. Intraoperative vital signs were as follows: blood pressure: 92-115/50-60 $\mathrm{mmHg}$, heart rate: $100-107 \mathrm{bpm}$, oxygen saturation: $100 \%$, and temperature: $36.3^{\circ} \mathrm{C}$. Propofol was stopped at the end of surgery. Following recovery of TOF to $90 \%$, neuromuscular blockade was reversed with pyridostigmine $4 \mathrm{mg}$ and glycopyrrolate $0.16 \mathrm{mg}$. The patient opened his eyes spontaneously, responded to verbal command, and was able to lift his head. He was subsequently extubated. Total anesthetic time was $45 \mathrm{~min}$. In the recovery room, muscular tone was re-evaluated but we did not check the TOF. Biopsy result confirmed Duchenne muscular dystrophy.

\section{Discussion}

DMD is the most common muscular dystrophy. It is a X-linked disease caused by mutations in the dystrophins gene. The disease is characterized by severe proximal muscle weakness, progressive degeneration, and fatty infiltration of the muscles. Serum creatine kinase is increased at the early stages, as do liver enzymes. The clinical course of DMD is severe and there is no therapy available. Progressive and severe muscle atrophy and weakness cause loss of ability to ambulate by the teenager. DMD affects the muscles of respiration and is associated with dilated cardiomyopathy, which often leads to death secondary to cardiopulmonary causes.

Patients with DMD are vulnerable to the adverse physiologic effects of general anesthesia and procedural sedation. They are at increased risk of rhabdomyolysis, life-threatening hyperkalemia, malignant hyperthermia-like reaction and heart failure when they are exposed to inhalational anesthetics and succinylcholine. Intravenous anesthetics such as propofol, ketamine and dexmedetomidine are reasonable alternatives and commonly used at variable doses [1-3]. However, there is no risk-free choice and a lack of prospective clinical studies to determine the safest anesthetic technique for DMD patients. In order to decrease risks such as rhabdomyolysis, we used small doses of midazolam and propofol under BIS monitoring.

The responsiveness of DMD patients administered with standard dosage of nondepolarizing neuromuscular blocking agent differs from that in normal patients. Delayed onset of blockade in DMD following administration of standard dose rocuronium, and the prolonged recovery from rocuroniuminduced blockade necessitate the need for careful assessment of neuromuscular function. If rocuronium is administered in DMD, assessment of complete neuromuscular recovery (TOF ratio $=0.9$ ) by quantitative measurement, such as acceleromyography, is mandatory.

However, use of reduced electrical current for pediatric TOF monitoring may be necessary [4-6]. Using succinylcholine for the setup and stabilization of neuromuscular monitoring in children has been reported [7]. However, this method is not appropriate for DMD. We used $30 \mathrm{~mA}$ at $2 \mathrm{~Hz}$ TOF during surgery. TOF was not established for the child in the recovery room, and we monitored the muscle strength clinically.

Anesthetic induction dosage of propofol decreases with increasing patient age. As such, bolus dose used for children are much higher than that of adult. According to the pharmacokinetics of child, initial loading dose and maintenance dose should be given more than adult $[8,9]$. Target-controlled infusion (TCI) system of propofol (Diprifusor ${ }^{\mathrm{TM}}$, Fresenius Vial SA, France) is based on adult pharmacokinetic model, and usage in children is limited [10]. The limitation of age and body weight for the TCI is 16 years-old and $30 \mathrm{~kg}$ respectively, and it is difficult to use in younger children. Our patient was only 6 years-old and weighted $19 \mathrm{~kg}$. Therefore, it was not appropriate to use TCI.

Bispectral index monitoring has been developed as a tool for measuring the depth of anesthesia in adults, but the use of BIS has generated several curious findings in children. The nervous system is functionally immature at birth, myelination is rapid during the first 2 years and completed by 7 years of age. Therefore, BIS cannot be applied to young children. And BIS increases with decreasing age $[11,12]$. Age itself is considered to be a factor affecting BIS values.

But some studies showed the validity of BIS in children older than 2 years [13-15]. Monitoring with BIS, not absolute value but trend is more important. BIS is the most valid monitor to measuring the depth of anesthesia to date. 
In conclusion, we recommend anesthetists to pay close attention to the neurophysiologic and neuromuscular monitoring for patients with Duchenne muscular dystrophic.

\section{References}

1. Shin HH, Park DH, Lee C, Woo SC, Kim YJ, Joo JH, et al. Anesthesia for a 10-year-old boy with Duchenne muscular dystrophy syndrome: A case report. Korean J Anesthesiol 2008; 55: 217-20.

2. Frankowski GA, Johnson JO, Tobias JD. Rapacuronium administration to two children with Duchenne's muscular dystrophy. Anesth Analg 2000; 91: 27-8.

3. Uslu M, Mellinghoff H, Diefenbach C. Mivacurium for muscle relaxation in a child with Duchenne's muscular dystrophy. Anesth Analg 1999; 89: 340-1.

4. Atlas G, Patel A. Pediatric-compatible train-of-four monitoring. Paediatr Anaesth 2005; 15: 707-8.

5. Tobias JD, Lynch A, McDuffee A, Garrett JS. Pancuronium infusion for neuromuscular block in children in the pediatric intensive care unit. Anesth Analg 1995; 81: 13-6.

6. Rodiera J, Serradell A, Alvarez-Gómez JA, Aliaga L. The cuff method: a pilot study of a new method of monitoring neuromuscular function. Acta Anaesthesiol Scand 2005; 49: 1552-8.

7. Withington D, Menard G, Varin F. Cisatracurium pharmacokinetics and pharmacodynamics during hypothermic cardiopulmonary bypass in infants and children. Paediatr Anaesth 2011; 21:341-6.

8. Aun CS, Short SM, Leung DH, Oh TE. Induction dose-response of propofol in unpremedicated children. Br J Anaesth 1992; 68: 64-7.

9. Marsh B, White M, Morton N, Kenny GN. Pharmacokinetic model driven infusion of propofol in children. Br J Anaesth 1991; 67: 41-8.

10. Han JI, Lee HS. Is it safe to use target-controlled propofol infusion with Diprifusor ${ }^{\mathrm{TM}}$ for children for school age? Korean J Anesthesiol 2004; 47: 667-70.

11. Oh AY, Gil NS, Yoon SJ, Kim HS, Kim SD, Kim CS. The differences in bispectral index according to age during sevoflurane anesthesia in children aged 1-7 years. Korean J Anesthesiol 2004; 47: 635-8.

12. Lerman J. Inhalation agents in pediatric anaesthesia - an update. Curr Opin Anaesthesiol 2007; 20: 221-6.

13. Degoute CS, Macabeo C, Dubreuil C, Duclaux R, Banssillon V. EEG bispectral index and hypnotic component of anaesthesia induced by sevoflurane: comparison between children and adults. Br J Anaesth 2001; 86: 209-12.

14. Davidson AJ, McCann ME, Devavaram P, Auble SA, Sullivan LJ, Gillis JM, et al. The differences in the bispectral index between infants and children during emergence from anesthesia after circumcision surgery. Anesth Analg 2001; 93: 326-30.

15. Bannister CF, Brosius KK, Sigl JC, Meyer BJ, Sebel PS. The effect of bispectral index monitoring on anesthetic use and recovery in children anesthetized with sevoflurane in nitrous oxide. Anesth Analg 2001; 92: 877-81. 